\title{
Anticoagulação oral nos muito idosos e seus determinantes clínicos
}

Márcia Christel Sá,* Maria João Balsa*

\section{RESUMO}

Objectivos: Determinar a frequência de anticoagulação nos idosos com 80 ou mais anos de idade inscritos numa USF, analisar factores associados à instituição desta terapêutica, caracterizar o subgrupo de doentes com fibrilação auricular quanto à pontuação nas escalas CHA2DS2VASc e HAS-BLED e analisar a prescrição antitrombótica respectiva.

Tipo de estudo: Estudo observacional analítico transversal.

Local: Unidade de Saúde Familiar Saúde em Família, Maia, Portugal.

População: Utentes inscritos nesta Unidade de Saúde Familiar com 80 ou mais anos de idade.

Métodos: Foi realizada uma colheita de dados dos processos electrónicos de uma amostra aleatória de 266 utentes. Foram registados idade e sexo do utente, prescrição de anticoagulantes orais e antiagregantes plaquetários, possíveis motivos clínicos e contra-indicações para anticoagulação, assim como patologias cardiovasculares associadas, último registo tensional e de índice de massa corporal.

Resultados: A frequência de anticoagulação foi de 6,8\% (IC95\%: 3,7-9,8\%). Da análise dos factores associados à anticoagulação, destacam-se com valores estatisticamente significativos os diagnósticos de fibrilação/flutter auricular, embolia pulmonar, doença arterial periférica, insuficiência cardíaca e enfarte agudo do miocárdio. Dos 31 utentes com fibrilação auricular, 35,5\% estavam anticoagulados, 9,7\% estavam anticoagulados e antiagregados, 45,2\% estavam antiagregados e 9,7\% não tinham terapêutica antitrombótica prescrita. Os utentes com esta arritmia tinham, segundo a escala de risco trombótico, indicação para anticoagulação.

Conclusões: A frequência de anticoagulação entre os utentes muito idosos desta unidade de saúde é elevada. A fibrilação auricular é o motivo provável da anticoagulação da maioria dos utentes. Grande parte dos indivíduos com esta arritmia encontrava-se sob terapêutica antitrombótica, mas a percentagem de utentes anticoagulados foi menor do que a esperada, de acordo com a ponderação das pontuações de risco trombótico e hemorrágico.

Palavras-chave: Anticoagulação; Muito Idoso.

\section{INTRODUÇÃO}

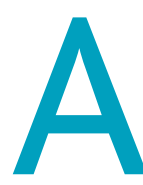

introdução da anticoagulação deve ser criteriosa, principalmente no idoso, tendo em conta os riscos e os benefícios da terapêutica. As principais indicações para anticoagulação oral são a presença de fibrilação auricular, doença valvular cardíaca ou tromboembolismo venoso (embolia pulmonar ou trombose venosa profunda). ${ }^{1,2}$ Para a profilaxia do tromboembolismo venoso, apesar da heparina de baixo peso molecular ser a opção mais válida, a

*Mestrado Integrado em Medicina (Faculdade de Medicina da Universidade do Porto). Internas de Formação Específica de Medicina Geral e Familiar. Unidade de Saúde Familiar Saúde em Família (Agrupamento de Centros de Saúde Grande Porto IV - Maia). anticoagulação oral pode ser considerada em diversas situações, nomeadamente artroplastia da anca ou do joelho, cirurgia de correcção de uma fractura da anca ou após um traumatismo major ou dano à medula espinal. $^{2}$

No caso da fibrilação auricular, a escala $\mathrm{CHA}_{2}$ $\mathrm{DS}_{2}$ VASc ajuda a estratificar os doentes consoante o seu risco trombótico, sendo recomendada a anticoagulação sempre que a pontuação seja superior a um. Assim, dado que a idade acima de 74 anos é cotada nesta escala com dois pontos, qualquer idoso nesta faixa etária com fibrilhação auricular terá indicação para ser anticoagulado. ${ }^{3}$ No entanto, pesa também o risco hemor- 
rágico, motivo de preocupação para o médico, o qual depende essencialmente da intensidade da anticoagulação e das características individuais do doente, nomeadamente da sua idade. ${ }^{2}$ Assim, a idade surge simultaneamente como um factor de risco trombótico e hemorrágico.

Os antagonistas da vitamina K aumentam o risco de hemorragia major em 0,3 a $0,5 \%$ ao ano e aumentam o risco de hemorragia cerebral em cerca de $0,2 \%$ ao ano, quando comparados com a ausência de tratamento anticoagulante. ${ }^{2}$ Alguns estudos mostram um aumento do risco hemorrágico em idosos com mais de 80 anos. No entanto, a intensidade da anticoagulação é um factor de risco mais importante do que a própria idade. ${ }^{2}$ Para ajudar o clínico na difícil decisão de anticoagular um doente, surgiram escalas de risco hemorrágico como o HEMORR2HAGES ${ }^{2}$ ou, no caso da fibrilhação auricular, o HAS-BLED. ${ }^{4}$

A frequência da anticoagulação não está ainda bem caracterizada na literatura. Um estudo finlandês, que envolveu 182091 habitantes, concluiu que a frequência de anticoagulação era de $0,69 \%$ para a população estudada, subindo para os $4,38 \%$ nos homens entre 75 e 79 anos e para $4,27 \%$ nas mulheres entre os 80 e 84 anos de idade; a média de idade dos doentes anticoagulados foi de 69 anos e a indicação mais frequente foi a fibrilação auricular (38\%), seguida da trombose venosa profunda (15\%) e da embolia pulmonar $(8 \%) .^{5}$

Um estudo sueco retrospectivo integrou registos electrónicos de 75146 indivíduos, concluindo que a frequência de anticoagulação era de $0,67 \%$, sendo maior no homem $(0,78 \%)$ do que na mulher $(0,58 \%)$; a média de idades da população anticoagulada foi de 72 anos; a faixa etária dos 75 aos 84 anos tinha uma frequência de $4,54 \%$. A principal indicação encontrada foi a fibrilação auricular $(41,6 \%)$, seguida da doença cerebrovascular (18,9\%), trombose venosa profunda (18,9\%) e prótese valvular cardíaca $(17,6 \%) .{ }^{6}$

Em Portugal não há estudos disponíveis nesta matéria, conhecendo-se apenas os dados relativos às vendas de medicamentos fornecidos pelo Observatório do Medicamento e Produtos de Saúde. Em Portugal Continental, no ano de 2003, foram vendidas 3,53 doses diárias definidas por 1000 habitantes por dia de antagonistas da vitamina $\mathrm{K}$, sendo 0,55 doses de acenocumarol e 2,98 doses de varfarina. ${ }^{7}$

\section{OBJECTIVOS}

O objectivo principal deste estudo foi determinar a frequência de anticoagulação e estudar a sua associação com aspectos clínicos da população de utentes com 80 ou mais anos de idade, inscritos na Unidade de Saúde Familiar (USF) Saúde em Família, Maia, Portugal.

Foram definidos como objectivos secundários os seguintes: determinar quais os anticoagulantes orais usados (varfarina ou acenocumarol); avaliar a presença de contra-indicações para instituição de terapêutica anticoagulante (hepatopatia, alcoolismo, alterações cognitivas, úlcera péptica, insuficiência renal, perturbações hemorrágicas, tensão arterial não controlada); determinar os fármacos antiagregantes usados; caracterizar o subgrupo de doentes com fibrilação auricular quanto à pontuação nas escalas $\mathrm{CHA}_{2} \mathrm{DS}_{2}$ VASc e HAS-BLED e analisar a prescrição antitrombótica respectiva.

\section{MÉTODOS}

Foi realizado um estudo observacional analítico transversal, na população de utentes inscritos na USF Saúde em Família (Agrupamento de Centros de Saúde Grande Porto IV - Maia) no ano de 2011, com 80 ou mais anos de idade ( $\mathrm{N}=609$ utentes). Através da aplicação informática Random ${ }^{\circledR}$, foi seleccionada uma amostra aleatória simples de 266 utentes. Esta dimensão amostral foi calculada com base na frequência de anticoagulação encontrada num estudo observacional sueco, na faixa etária dos 75 aos 84 anos $(4,54 \%),{ }^{6}$ para um nível de significância de $5 \%$ e erro amostral admitido de $2,5 \%$.

Foi revisto o processo clínico electrónico de cada utente através do Sistema de Apoio ao Médico (SAM) e recolhida informação relativa às seguintes variáveis: idade, sexo, últimos valores de tensão arterial e de índice de massa corporal (IMC), fibrilação auricular/flutter auricular (K78), embolia pulmonar ou trombose venosa profunda (K93), prótese valvular mecânica (K83) e prótese de anca ou joelho (A89), cardiopatia isquémica (K74, K76), enfarte agudo do miocárdio (K75), insuficiência cardíaca (K77), hipertensão arterial (K86, K87), acidente isquémico transitório (K89), acidente vascular cerebral (K90, K91), doença arterial periférica (K92) e diabetes mellitus (T89, T90). A obesidade foi considerada se registo de IMC superior a $30 \mathrm{~kg} / \mathrm{m}^{2}$. As patologias em estudo foram consideradas se estives- 
sem registadas no campo dos «Problemas» ou nos «Antecedentes» do processo clínico. A fibrilação auricular foi também considerada se existisse registo da sua presença em electrocardiograma no campo dos meios complementares de diagnóstico. Foi usada a Classificação Internacional de Cuidados Primários - 2. ${ }^{a}$ edição ${ }^{8}$ (ICPC-2) para registo das patologias.

$\mathrm{O}$ utente foi considerado anticoagulado se tivesse prescrição prévia de anticoagulantes orais (varfarina ou acenocumarol) no receituário electrónico entre 2008 e 2011, ou se estivesse registada a sua condição de anticoagulado no campo das «Observações» do processo clínico informatizado. Foi também registada a prescrição de antiagregantes plaquetários (ácido acetilsalicílico, clopidogrel, triflusal, ticlopidina ou dipiridamol).

Como contra-indicações para anticoagulação foram ainda pesquisadas no processo clínico os seguintes diagnósticos: úlcera péptica (D86), doença hepática (D97), alcoolismo (P15), alterações cognitivas (P28, P70, P85, N99), insuficiência renal (U99) e perturbações hemorrágicas (A10, D14, D15, D16, F75, H05, R24, U06).

No subgrupo dos doentes com fibrilação auricular as variáveis estudadas permitiram ainda o cálculo da pontuação das escalas $\mathrm{CHA}_{2} \mathrm{DS}_{2} \mathrm{VASc}$ - escala de risco trombótico que contempla insuficiência cardíaca, hipertensão arterial, idade acima de 74 anos, diabetes mellitus, acidente vascular cerebral ou acidente isquémico transitório prévios, doença vascular periférica, idade entre 65 e 74 anos, sexo feminino; e HAS-BLED - escala de risco hemorrágico que contempla hipertensão arterial, idade acima de 64 anos, acidente vascular cerebral prévio, perturbações hemorrágicas, alcoolismo, função renal ou hepáticas anormais. O INR lábil e o uso de medicação concomitante entraram para o cálculo desta pontuação como zero, visto não terem sido recolhidos esses dados na amostra estudada.

A análise dos dados foi realizada com recurso ao programa informático Microsoft Excel $2010 \AA$, sendo as variáveis categóricas apresentadas em número absoluto e percentagem e as variáveis numéricas em média e desvio-padrão. A análise bivariada foi efectuada com base nos testes estatísticos t de student para comparação de variáveis contínuas, nomeadamente a idade, e Qui-quadrado e Teste de Fisher para comparação de variáveis categóricas.

A realização deste estudo teve parecer favorável da
Comissão de Ética para a Saúde da Administração Regional da Saúde do Norte, tendo sido aprovado pelo ACES Grande Porto IV - Maia e pelos elementos da USF Saúde em Família.

\section{RESULTADOS}

Foram avaliados os registos de 266 utentes, sendo que 238 (89,5\%) eram utilizadores da consulta, ou seja, tiveram pelo menos uma consulta presencial com o seu médico de família desde a sua inscrição na USF Saúde em Família.

Os dados demográficos foram apurados em todos os utentes. Os valores de tensão arterial foram apurados em apenas 201 utentes e os valores de IMC em 158 utentes. Os restantes utentes que não tinham estes registos não foram excluídos do estudo, visto apresentarem dados relativos a todos os outros parâmetros. A análise descritiva dos dados obtidos na amostra encontra-se apresentada no Quadro I.

A frequência de anticoagulação foi de 6,8\% [IC95\%: $(3,7-9,8)]$ no total da amostra e de 7,6\% [IC95\%: $(4,2-$ $10,9)]$ se considerados apenas os utilizadores da consulta.

\begin{tabular}{|l|c|c|}
\hline \multicolumn{2}{|c|}{ QUADRO I. Caracterização da amostra $(\mathbf{n}=266)$. } \\
\hline idade (em anos) & $\begin{array}{c}\text { desvio } \\
\text { padrão }\end{array}$ \\
\hline & 85,5 & 4,7 \\
\hline sexo masculino & & \\
\hline hipertensão arterial & $\mathbf{n}$ & $\%$ \\
\hline diabetes mellitus & 84 & 31,6 \\
\hline doença arterial periférica & 138 & 51,9 \\
\hline insuficiência cardíaca & 48 & 18,0 \\
\hline acidente vascular cerebral & 38 & 4,9 \\
\hline acidente isquémico transitório & 7 & 14,7 \\
\hline enfarte agudo do miocárdio & 5 & 14,3 \\
\hline cardiopatia isquémica & 13 & 2,6 \\
\hline obesidade & 35 & 13,2 \\
\hline
\end{tabular}


Foram identificados 77 (32,4\%) doentes medicados com antiagregantes plaquetários. No quadro II encontram-se resumidas as frequências por fármacos antitrombóticos usados, destacando-se a varfarina no grupo dos anticoagulantes e o ácido acetilsalicílico nos antiagregantes.

Nos Quadros III e IV encontram-se os resultados da análise dos factores associados à anticoagulação. Destacam-se com valores estatisticamente significativos os diagnósticos de fibrilação/flutter auricular, embolia pulmonar, doença arterial periférica, insuficiência cardíaca e enfarte agudo do miocárdio.

Estudou-se a relação entre anticoagulação e problemas de saúde que constituem contra-indicações para a sua implementação. Os resultados encontram-se no Quadro V e nenhuma diferença apresentou significância estatística.

Dos 31 utentes com fibrilhação auricular, 14 (45,2\%) estavam anticoagulados, 11 (35,5\%) estavam antiagregados, três $(9,7 \%)$ estavam anticoagulados e antiagregados, e três $(9,7 \%)$ não tinham terapêutica antitrombótica prescrita.

Foram calculados o risco trombótico e o risco hemorrágico pela pontuação das escalas $\mathrm{CHA}_{2} \mathrm{DS}_{2} \mathrm{VASc} \mathrm{e}$ HAS-BLED, respectivamente, para os utentes com fibrilação auricular-Quadro VI. Os três utentes com esta arritmia e sem qualquer terapêutica antitrombótica apresentavam pontuações de risco trombótico entre três e oito sendo que a respectiva pontuação na escala de risco hemorrágico foi de um.

\section{DISCUSSÃO}

A frequência de anticoagulação por nós obtida situou-se nos 6,8\% para o total da amostra e nos 7,6\% entre os utilizadores da consulta, valores superiores aos encontrados noutros estudos $-4,5 \%$ num estudo sueco retrospectivo entre utentes na faixa etária dos 75 aos 84 anos $^{6}$ e 4,4\% nos homens entre 75 e 79 anos e 4,3\% nas mulheres entre os 80 e 84 anos de idade de um estudo finlandês. ${ }^{5}$

Esta elevada frequência de anticoagulação obtida no nosso estudo pode em parte dever-se à metodologia utilizada - foram considerados como anticoagulados os indivíduos em cujo processo clínico constava a prescrição de um anticoagulante oral em qualquer momento entre

\begin{tabular}{|c|c|c|c|}
\hline \multicolumn{4}{|c|}{$\begin{array}{l}\text { QUADRO II. Fármacos anticoagulantes e } \\
\text { antiagregantes utilizados. }\end{array}$} \\
\hline & fármaco & $\mathbf{n}$ & $\%$ \\
\hline \multirow[t]{2}{*}{ anticoagulação } & varfarina & 12 & 66,7 \\
\hline & acenocumarol & 6 & 33,3 \\
\hline \multirow[t]{5}{*}{ antiagregação } & ácido acetilsalicílico & 52 & 67,5 \\
\hline & clopidogrel & 12 & 15,6 \\
\hline & triflusal & 2 & 2,6 \\
\hline & ticlopidina & 2 & 2,6 \\
\hline & > 1 antiagregante & 9 & 11,7 \\
\hline
\end{tabular}

2008 e 2011, pelo que poderão ter sido incluídos utentes nos quais a anticoagulação tenha entretanto sido suspensa, nomeadamente se houve desenvolvimento de novos factores de risco para hemorragia ou interrupção da necessidade da mesma - frequente no caso da profilaxia de tromboembolismo.

Por outro lado, reconhecemos que alguns utentes poderão estar anticoagulados e não terem sido classificados como tal, na medida em que a anticoagulação pode nunca ter sido prescrita ou registada pelo médico de família.

Os utentes do sexo masculino apresentaram maior frequência de anticoagulação $(9,2 \%)$ que os do sexo feminino $(6,8 \%)$. Esta diferença não foi estatisticamente significativa, o que poderá dever-se a um erro tipo II pois diferenças efectivas entre os dois sexos foram observadas em dois outros estudos suecos. ${ }^{6,9} \mathrm{O}$ facto da fibrilação auricular ter sido o principal motivo de hipocoagulação no presente estudo poderá explicar a maior frequência de hipocoagulação no sexo masculino, pois
QUADRO III. Análise bivariada de factores associados a anticoagulação (variável quantitativa).

\begin{tabular}{l|r|c|c|c} 
& & \multicolumn{2}{|c|}{ idade } & \\
\hline \multirow{2}{*}{ anticoagulação } & & média & desvio padrão & $\mathrm{P}^{*}$ \\
\cline { 2 - 5 } & não $(\mathrm{n}=220)$ & 86,2 & 4,8 & 0,14 \\
\hline
\end{tabular}

*Teste $\mathrm{t}$ de student 


\begin{tabular}{|c|c|c|c|c|}
\hline & & $\mathrm{n}$ & $\begin{array}{c}\text { \% de } \\
\text { anticoagulação }\end{array}$ & $\mathbf{p}$ \\
\hline \multirow[t]{2}{*}{ sexo } & masculino & 76 & 9,2 & \multirow[t]{2}{*}{$0,51^{*}$} \\
\hline & feminino & 162 & 6,8 & \\
\hline \multirow[t]{2}{*}{ FA/flutter auricular } & $\operatorname{sim}$ & 31 & 45,0 & \multirow[t]{2}{*}{$<0,001^{*}$} \\
\hline & não & 207 & 1,9 & \\
\hline \multirow[t]{2}{*}{ prótese valvular mecânica } & $\operatorname{sim}$ & 1 & 0,0 & \multirow[t]{2}{*}{$1 * *$} \\
\hline & não & 237 & 7,6 & \\
\hline \multirow[t]{2}{*}{ embolia pulmonar } & $\operatorname{sim}$ & 4 & 50,0 & \multirow[t]{2}{*}{$0,03 * *$} \\
\hline & não & 234 & 6,8 & \\
\hline \multirow[t]{2}{*}{ doença arterial periférica } & $\operatorname{sim}$ & 13 & 23,1 & \multirow[t]{2}{*}{$0,03^{*}$} \\
\hline & não & 225 & 6,7 & \\
\hline \multirow[t]{2}{*}{ insuficiência cardíaca } & $\operatorname{sim}$ & 39 & 25,6 & \multirow[t]{2}{*}{$<0,001^{*}$} \\
\hline & não & 199 & 4,0 & \\
\hline \multirow{2}{*}{ acidente vascular cerebral } & $\operatorname{sim}$ & 38 & 5,2 & \multirow[t]{2}{*}{$0,56^{*}$} \\
\hline & não & 200 & 8,0 & \\
\hline \multirow[t]{2}{*}{ acidente isquémico transitório } & $\operatorname{sim}$ & 7 & 0,0 & \multirow[t]{2}{*}{$1^{* *}$} \\
\hline & não & 231 & 7,7 & \\
\hline \multirow[t]{2}{*}{ enfarte agudo do miocárdio } & $\operatorname{sim}$ & 5 & 40,0 & \multirow[t]{2}{*}{$0,047^{* *}$} \\
\hline & não & 233 & 6,9 & \\
\hline \multirow[t]{2}{*}{ cardiopatia isquémica } & $\operatorname{sim}$ & 13 & 15,4 & \multirow[t]{2}{*}{$0,27^{*}$} \\
\hline & não & 225 & 7,1 & \\
\hline \multirow{2}{*}{ hipertensão arterial } & $\operatorname{sim}$ & 138 & 8,0 & \multirow[t]{2}{*}{$0,78^{*}$} \\
\hline & não & 100 & 7,0 & \\
\hline \multirow[t]{2}{*}{ diabetes mellitus } & $\operatorname{sim}$ & 48 & 8,3 & \multirow[t]{2}{*}{$0,82 *$} \\
\hline & não & 190 & 7,3 & \\
\hline
\end{tabular}

* Teste de Qui-quadrado; ** Teste exacto de Fisher

esta patologia foi mais frequente em homens $(15,5 \%)$ que em mulheres $(9,9 \%)$.

A modalidade de registo no SAM não nos permite afirmar taxativamente que determinado factor constituiu o motivo da anticoagulação do utente, apenas o podemos inferir a partir da sua coexistência, limitação igualmente inerente à metodologia usada no estudo (estudo transversal). De referir ainda que a indicação para anticoagulação «uso de prótese valvular cardíaca» não é passível de codificação directa pelo ICPC-2. As- sim sendo, utilizámos por aproximação o código K83 - doença valvular cardíaca, que inclui outras alterações valvulares que não necessariamente protésicas. Assim, a existência de válvula mecânica, que neste estudo foi o motivo provável de anticoagulação de um utente, pode não corresponder à indicação para o início desta terapêutica. Existem ainda outros possíveis motivos para a realização de anticoagulação, como por exemplo a existência de coagulopatias que optámos por não incluir no estudo, dada a sua reduzida prevalência comparativamente às patologias estudadas e ao facto da sua codificação não ser possível com recurso à ICPC-2.

A associação estatisticamente significativa entre anticoagulação e fibrilação/flutter auricular e embolia pulmonar é concordante com a bibliografia: num trabalho recente realizado nos Estados Unidos da América, a nível hospitalar, a principal indicação para anticoagulação foi a fibrilação auricular (46\%) seguida do tromboembolismo (34\%) e só depois a existência de uma válvula mecânica (14\%);11 num outro estudo realizado em França, também de cariz hospitalar, mas já numa população seleccionada de indivíduos com 70 ou mais anos, a principal indicação foi fibrilação auricular (69\%) seguida do tromboembolismo (28\%): ${ }^{12}$ num estudo finlandês, a principal indicação foi igualmente a existência de fibrilação auricular (38\%) e a segunda indicação mais frequente tratou-se da trombose venosa profunda (15\%). ${ }^{5}$

Existem outros trabalhos que apresentam resultados diferentes: a indicação mais comum num estudo realizado na Suécia foi o $\mathrm{AVC}^{9} \mathrm{e}$, num estudo escocês ${ }^{10}$, 


\begin{tabular}{|c|c|c|c|c|}
\hline \multicolumn{5}{|c|}{$\begin{array}{l}\text { QUADRO V. Relação entre existência de contra-indicações e } \\
\text { frequência de anticoagulação. }\end{array}$} \\
\hline & & & $\begin{array}{l}\text { \% de } \\
\text { anticoagulação }\end{array}$ & $\mathbf{P}$ \\
\hline \multirow[t]{2}{*}{ doenças do fígado } & $\operatorname{sim}$ & 7 & 28,6 & \multirow[t]{2}{*}{$0,09 * *$} \\
\hline & não & 231 & 6,9 & \\
\hline \multirow[t]{2}{*}{ abuso crónico do álcool } & $\operatorname{sim}$ & 1 & 0,0 & \multirow[t]{2}{*}{$1 * *$} \\
\hline & não & 237 & 7,6 & \\
\hline \multirow{2}{*}{ alterações cognitivas } & $\operatorname{sim}$ & 23 & 8,7 & \multirow[t]{2}{*}{$0,69 * *$} \\
\hline & não & 215 & 7,4 & \\
\hline \multirow[t]{2}{*}{ úlcera péptica } & $\operatorname{sim}$ & 1 & 0,0 & \multirow[t]{2}{*}{$1 * *$} \\
\hline & não & 237 & 7,6 & \\
\hline \multirow[t]{2}{*}{ insuficiência renal } & $\operatorname{sim}$ & 5 & 20,0 & \multirow[t]{2}{*}{$0,33^{* *}$} \\
\hline & não & 233 & 7,3 & \\
\hline \multirow[t]{2}{*}{ perturbações hemorrágicas } & $\operatorname{sim}$ & 8 & 25,0 & \multirow[t]{2}{*}{$0,12^{* *}$} \\
\hline & não & 230 & 7,0 & \\
\hline \multirow[t]{2}{*}{$\mathrm{TA} \geq 140 / 90 \mathrm{mmHg}$} & $\operatorname{sim}$ & 88 & 6,8 & \multirow[t]{2}{*}{$0,6^{*}$} \\
\hline & não & 113 & 8,8 & \\
\hline
\end{tabular}

* Teste de Qui-quadrado; ** Teste exacto de Fisher

entre os utentes anticoagulados de todas as idades seguidos pelo seu médico de família, $34 \%$ iniciaram terapêutica anticoagulante por apresentar válvula protésica; o tromboembolismo levou à anticoagulação de $13 \%$ dos utentes e a fibrilação auricular foi a indicação para anticoagulação em apenas $9 \%$ dos doentes.

No estudo finlandês já citado verificou-se que os motivos que levaram ao início da terapêutica anticoagulante variaram consoante a idade, tendo os utentes mais novos a trombose venosa profunda como principal indicação (utentes estes com uma idade média de 65,8 anos). Por outro lado, sabe-se que a prevalência de fibrilação auricular aumenta com a idade, ${ }^{3}$ o que pode explicar parcialmente a existência de mais doentes anticoagulados por fibrilação auricular no nosso estudo.

Dos 31 utentes com fibrilação auricular, 17 (54,8\%) estão antiagregados, $14(45,2 \%)$ estão antiacoagulados e três (9,7\%) não têm terapêutica antitrombótica prescrita. Admitimos que possa haver utentes classificados como não anticoagulados/antiagregados e que podiam na realidade estar a realizar esta terapêutica sem que tal se encontre assinalado no processo clínico, pelos motivos já apresentados. No que diz respeito à antiagregação, acresce ainda o facto de alguns destes fármacos não serem comparticipados, o que pode contribuir para ausências de registo da sua prescrição.

Entre os utentes com fibrilação auricular, dois apresentam alterações cognitivas e cinco apresentam valores tensionais iguais ou superiores a 140/90 mmHg. Estas condições podem eventualmente ter sido consideradas como contra-indicação relativa à anticoagulação, pois estes utentes encontravam-se antiagregados.

A idade avançada é por vezes apresentada como argumento para a utilização de terapêutica antiagregante em detrimento da anticoagulante. ${ }^{12}$ No entanto, há estudos que mostram que, com o avançar dos anos, a

QUADRO VI. Terapêutica antitrombótica segundo a pontuação das escalas CHA2DS2VASc e HAS-BLED nos utentes com fibrilhação auricular.

\begin{tabular}{l|c|c|c|c|c} 
& pontuação & $\begin{array}{c}\text { anticoagulação } \\
(\mathbf{n}=14)\end{array}$ & $\begin{array}{c}\text { antiagregação } \\
(\mathbf{n}=17)\end{array}$ & $\begin{array}{c}\text { e antiagregação } \\
(\mathbf{n}=\mathbf{3})\end{array}$ & $\begin{array}{c}\text { sem terapia } \\
\text { antitrombótica } \\
(\mathbf{n}=3)\end{array}$ \\
\hline CHA $_{2}$ DS 2 VASC & $\leq 1$ & 0 & 0 & 0 & 0 \\
\cline { 2 - 6 } & $>1$ & 14 & 17 & 3 & 3 \\
\hline HAS-BLED & $\leq 3$ & 13 & 15 & 2 & 3 \\
\cline { 2 - 6 } & $>3$ & 1 & 2 & 1 & 0
\end{tabular}


eficácia relativa da primeira modalidade terapêutica para a prevenção de acidente vascular cerebral diminui, mantendo-se inalterada para a segunda. ${ }^{3}$ Acresce ainda o facto de alguns estudos, entre eles o estudo WAS$\mathrm{PO},{ }^{13}$ realizado igualmente numa população de 75 octogenários com fibrilação auricular, terem verificado um maior número de eventos adversos (nomeadamente hemorragia grave) com o uso de ácido acetilsalicílico (33\%) comparativamente à varfarina $(6 \%)$. De referir ainda o estudo ACTIVE $\mathrm{W},{ }^{14}$ realizado numa população de 6706 utentes com mais de 74 anos com fibrilação auricular, que mostrou que o ramo da investigação com terapia anticoagulante foi superior à combinação de clopidogrel e ácido acetilsalicílico na prevenção de eventos vasculares, sem diferenças estatisticamente significativas no que diz respeito a eventos hemorrágicos.

A escala $\mathrm{CHA}_{2} \mathrm{DS}_{2}$ VASc ajuda a estratificar os doentes com fibrilação auricular de acordo com o seu risco trombótico. ${ }^{3}$ Os utentes com esta arritmia incluídos no nosso estudo, apenas pelo facto de apresentarem idade superior a 79 anos, somam uma pontuação igual ou superior a dois, estando por isso recomendada a instituição de anticoagulação.

Três participantes com fibrilação auricular não se encontravam sob qualquer terapêutica antitrombótica. Estes utentes apresentavam pontuações no score $\mathrm{CHA}_{2} \mathrm{DS}_{2}$ VASc entre três e oito; a pontuação obtida por estes mesmos utentes na escala HAS-BLED (escala de risco hemorrágico) é igual a um em todos eles. Assim, de acordo com as mais recentes orientações sobre fibrilação auricular da Sociedade Europeia de Cardiologia, mesmo estes utentes teriam eventualmente indicação para realizar terapêutica anticoagulante. De referir, no entanto, que a pontuação da escala HAS-BLED foi calculada sem ter em conta o uso concomitante de outras medicações nem com os valores de INR lábeis, o que poderia elevar esta pontuação. No entanto, o valor máximo que poderia ser atingido, caso estes dois parâmetros não contemplados estivessem presentes, seria de três, precisamente o limite a partir do qual se considera haver risco hemorrágico e ser necessária uma vigilância mais apertada após a iniciação de terapêutica antitrombótica, não contraindicando no entanto a sua instituição. ${ }^{3}$

De realçar que muitas vezes o doente individual não se enquadra nos parâmetros definidos pelas orientações e por isso a ponderação entre o risco e o benefício da melhor opção terapêutica para cada doente cabe ao seu médico, estando este na posição privilegiada de conhecer o contexto específico de cada um dos seus utentes. É necessário ter em conta as preferências do próprio utente e saber se se encontram reunidas condições para um cumprimento adequado da terapêutica e controlo do INR, factores que poderão igualmente ser a justificação para estes três utentes não estarem anticoagulados ou para outros 17 com fibrilação auricular estarem apenas antiagregados.

Para além das limitações já referidas, gostaríamos de salientar que, da amostra seleccionada, apenas 238 indivíduos (89,5\%) apresentavam registos no seu processo clínico. Dada a idade avançada dos sujeitos estudados, alguns destes utentes residem em lares, tendo acompanhamento médico nestas instituições, não fazendo uso dos serviços prestados na USF. A razão pela qual estes utentes se encontram institucionalizados pode eventualmente associar-se ao facto de apresentarem maior número de comorbilidades, pelo que a prevalência de determinadas patologias por nós apresentada pode estar subestimada, dada a ausência de registos no processo clínico da USF. Além disso, mesmo entre os utilizadores, admitimos que a codificação de patologias e o registo de medicação antitrombótica em curso possam estar subestimados. Por fim, o facto de o estudo ter sido conduzido apenas numa Unidade de Saúde pode limitar a generalização dos resultados.

Em conclusão, a frequência de anticoagulação entre os utentes muito idosos desta unidade de saúde é elevada. A fibrilação auricular é o motivo provável da anticoagulação da maioria dos utentes. Grande parte dos indivíduos com esta arritmia encontrava-se sob terapêutica antitrombótica, mas a percentagem de utentes anticoagulados foi menor do que a esperada, de acordo com a ponderação das pontuações de risco trombótico e hemorrágico. A existência de alterações cognitivas, mau controlo tensional ou a própria preferência do utente podem ter contribuído para esse facto.

Com o aumento progressivo da esperança média de vida e consequente aumento do número de utentes a cargo do médico de família com indicação para anticoagulação, é recomendável a elaboração de estratégias para uma abordagem sistematizada ao tratamento an- 
ticoagulante, maximizando a adesão às orientações de anticoagulação pelos prestadores de Cuidados de Saúde Primários, sem nunca esquecer o dever de adequação de qualquer procedimento às necessidades do utente individual.

No futuro, a introdução de novos fármacos anticoagulantes no mercado, com diferente perfil de segurança, comodidade e eficácia poderá substituir o arsenal medicamentoso actualmente disponível e revolucionar esta área terapêutica.

\section{REFERÊNCIAS BIBLIOGRÁFICAS:}

1. Baglin TP, Keeling DM, Watson HG; British Committee for Standards in Haematology. Guidelines on oral anticoagulation (warfarin): third edition - 2005 update. Br J Haematol 2006 Feb; 132 (3): 277-85.

2. Robert-Ebadi H, Le Gal G, Righini M. Use of anticoagulants in elderly patients: practical recommendations. Clin Interv Aging 2009; 4: 16577.

3. Camm AJ, Kirchhof P, Lip GY, Schotten U, Savelieva I, Ernst S, et al. Guidelines for the management of atrial fibrillation: the Task Force for the Management of Attrial Fibrillation of the European Society of Cardiology (ESC). Eur Heart J 2010 Oct; 31 (19): 2369-429.

4. Pisters R, Lane DA, Nieuwlaat R, de Vos CB, Crijns HJ, Lip GY. A novel user-friendly score (HAS-BLED) to assess one-year risk of major bleeding in patients with atrial fibrillation: the Euro Heart Survey. Chest 2010 Nov; 138 (5): 1093-100.

5. Eskola K, Aittoniemi P, Kurunmaki H, Latva-Necvala A, Paloneva M, Wallin AM, et al. Anticoagulant treatment in primary health care in Finland. Scand J Prim Health Care 1996 Sep; 14 (6): 165-70.

6. Nilsson $\mathrm{GH}$, Bjorholt I, Johnsson H. Anticoagulant treatment in primary health care in Sweden - prevalence, incidence and treatment diagnosis: a retrospective study on electronic patient records in a registered population. BMC Fam Pract 2003 Apr 1; 4: 3.

7. Furtado C. Análise da evolução da utilização dos anticoagulantes e antitrombóticos em Portugal Continental entre 1999 e 2003. Lisboa: Observatório do Medicamento e Produtos de Saúde; 2005.
8. World Organizations of National Colleges, Academies, and Academic Associations of General Practitioners / Family Physicians (WONCA). ICPC-2: International Classification of Primary Care. 2nd ed. Oxford, NY: Oxford University Press; 1998.

9. Wandell PE. Anticoagulant patients in Swedish primary health care: a comparison 5 years apart. Scand J Prim Health Care 1998 Sep; 16 (3): 183-7.

10. Pell JP, Mclver B, Stuart P, Malone DN, Alcock J. Comparison of anticoagulant control among patients attending general practice

11. and a hospital anticoagulant clinic. Br J Gen Pract 1993 Apr; 43 (369): $152-4$.

12. Dawson NL, Klipa D, O'Brien AK, Crook JE, Cucchi MW, Valentino AK. Oral anticoagulation in the hospital: analysis of patients at risk. JThromb Thrombolysis 2011 Jan; 31 (1): 22-6.

13. Gouin-Thibault I, Levy C, Pautas E, Cambus JP, Drouet L, Mahé I, et al. Improving anticoagulation control in hospitalized elderly patients on warfarin. J Am Geriatr Soc 2010 Feb; 58 (2): 242-7.

14. Rash A, Downes T, Portner R, Yeo WW, Morgan N, Channer KS. A randomised controlled trial of warfarin versus aspirin for stroke prevention in octogenarians with atrial fibrillation (WASPO). Age Ageing 2007 Mar; 36 (2): 151-6.

15. Connolly S, Pogue J, Hart RG, Pfeffer M, Hohnloser S, Chrolavicius S, et al. Clopidogrel plus aspirin versus oral anticoagulation for atrial fibrillation in the Atrial fibrillation Clopidogrel Trial with Irbesartan for prevention of Vascular Events (ACTIVE W): a randomised controlled trial. Lancet 2006 Jun 10; 367 (9526): 1903-12.

\section{CONFLITOS DE INTERESSE}

As autoras declaram não existir qualquer tipo de conflitos de interesse.

\section{ENDEREÇO PARA CORRESPONDÊNCIA}

Márcia Christel de Carvalho Sá

Rua Grupo Desportivo de Águas Santas, n. ${ }^{\circ} 33,1{ }^{\circ}$ Direito Frente

4425-096 Maia

E-mail: christelsa@gmail.com

Recebido em 12/08/2011

Aceite para publicação em 27/06/2012 


\section{ABSTRACT}

\section{ORAL ANTICOAGULATION THERAPY IN ELDERLY PATIENTS}

Objectives: To determine the frequency of anticoagulation therapy among patients over 80 years of age registered in a Family Health Unit, to analyze factors associated with this treatment, and to characterize patients with atrial fibrillation using the CHA2DS2VASC and HAS-BLED scales.

Type of study: Cross-sectional.

Location: Maia Family Health Unit, Portugal.

Population: All patients over 80 years old registered in this Family Health Unit.

Methods: We studied the electronic medical records of a random sample of elderly patients. We recorded the patients' age, gender, prescription of oral anticoagulants and antiplatelet agents, indications and contraindications for anticoagulation, the presence of cardiovascular diseases, blood pressure and body mass index.

Results: The records of 266 patients were selected for study out of the 609 records of patients over 80 registered in this clinic. The frequency of anticoagulation was $6.8 \%$ (95\% Cl: 3.7 to 9.8\%). Anticoagulation was associated with the diagnosis of atrial fibrillation, pulmonary embolism, peripheral arterial disease, heart failure and acute myocardial infarction. Of the 31 patients with atrial fibrillation, 35.5\% were receiving anticoagulation therapy, 9.7\% had both anticoagulation and antiplatelet therapy, $45.2 \%$ used antiplatelet therapy and $9.7 \%$ were not receiving any antithrombotic therapy. All patients with atrial fibrillation had indications for anticoagulation.

Conclusions: The frequency of anticoagulation among very elderly patients in this family health unit is high. Atrial fibrillation was the most common indication. Most individuals with atrial fibrillation were receiving antithrombotic therapy but the proportion of anticoagulant users was less than expected, based on the use of thrombosis and bleeding risk scales.

Key-words: Anticoagulants; Aged 80 and Over. 